\title{
Educación para la paz. Una mirada hacia el modelo para Jalisco
}

\author{
Education to make peace. \\ A look to Jalisco Model
}

\begin{abstract}
Teresa Magnolia Preciado Rodríguez
Doctora en Derecho Electoral por el Instituto Prisciliano Sánchez del Tribunal Electoral del Estado de Jalisco, Profesora de Tiempo Completo de la Universidad de Guadalajara adscrita al Centro Universitario de Tonalá, Coordinadora del Doctorado en Derechos Humanos. Correo electrónico: magnolñia.preciado@academicos.udg.mx
\end{abstract}

RESUMEN: La educación en México ha cambiado en las últimas décadas: según datos estadísticos proporcionados por la INEGI, desde 1980 hasta la fecha e acuerdo el porcentaje de población de 6 a 14 años que asisten a la escuela ha aumentado. Sin embargo, los números entre las entidades del país no son homogéneos.

Los estados que presentan las cifras más bajas de jóvenes en las escuelas -entre $93 \%$ y $95.9 \%$ - son Chihuahua, Jalisco, Colima, Michoacán, Guanajuato, Guerrero, Puebla, Veracruz, Oaxaca, Chiapas y Campeche. La encuesta arroja que los municipios con la menor cantidad de población en escuelas son Riva Palacio (Chihuahua), San Simón Zahuatlán (Oaxaca) y Batopilas (Chihuahua).

Los estados que presentan una población de entre 6 y 14 años mayor al 97\% en las escuelas son Baja California Sur, Sinaloa, Nuevo León, San Luis Potosí, Queré-
ABSTRACT: The states that present the lowest numbers of young people in schools -between $93 \%$ and $95.9 \%-$ are Chihuahua, Jalisco, Colima, Michoacán, Guanajuato, Guerrero, Puebla, Veracruz, Oaxaca, Chiapas and Campeche. The survey shows that the municipalities with the least population in schools are Riva Palacio (Chihuahua), San Simón Zahuatlán (Oaxaca) and Batopilas (Chihuahua).

The states that present a population between 6 and 14 years of age greater than $97 \%$ in schools are Baja California Sur, Sinaloa, Nuevo León, San Luis Potosí, Querétaro, Hidalgo, State of Mexico, Mexico City and Yucatán.

For this work we will focus on Jalisco, being one of the states with the worst percentage of young people conducting studies, this study will focus on the analysis of the educational model that the entity proposes. The objective of this work Recibido: 15 de junio de 2020. Dictaminado: 03 de julio de 2020. 
taro, Hidalgo, Estado de México, Ciudad de México y Yucatán.

Para este trabajo nos enfocaremos en Jalisco, siendo uno de los estados con peor porcentaje de jóvenes realizando estudios, este estudió se enfocará en el análisis del modelo educativo que la entidad propone. Este trabajo tiene como objetivo comparar el trabajo que al día de hoy se realiza, en comparación a los modelos que son utilizados en el mundo $\mathrm{y}$ otros estados.

Palabras Clave: Modelo, Educación, Garantía, Obligación, Derechos Humanos, presupuesto, financiamiento, Organización internacional is to compare with that is done today, in comparison to the models that are used in the world and other states.

Keywords: Model, Education, Guarantee, Obligation, Human Rights, budget, financing, International organization

SUMARIO: I. INTRODUCCIÓN; II. GENERALIDADES DEL CONTEXTO; III. MARCO JURÍDICO; IV. DEBATE ACTUAL; EDUCACIÓN EN DERECHOS HUMANOS PARA LOGRAR PAZ V. CONCLUSIONES; VI. BIBLIOGRAFÍA.

\section{Introducción}

La educación es sin duda la clave para muchos de los grandes problemas de nuestra sociedad. Como derecho humano, la educación es lo que llamamos un derecho llave, un derecho que permite el acceso y garantía de otros derechos, trabajo, alimento, salud, cultura, desarrollo personal, la paz, entre otros. Sin embargo, es precisamente uno de los derechos en los que vemos mayor desigualdad. Las brechas sociales acrecientan la problemática de acceso a la educación, y se agudizan según la región, según los grupos vulnerables.

Como lo he señalado en trabajos anteriores, los discursos oficiales de cualquier nación afirman que el respeto a los derechos humanos 
es la base de todos los actos del gobierno; en los organismos internacionales la retórica supone como un hecho, real y tangible el avance en materia de protección de derechos humanos; incluso el engranaje constitucional -nacional y supranacional- es básicamente perfecto en cuanto al diseño legislativo para al reconocimiento y garantía de cualquier derecho humano, incluyendo claro la educación, sin embargo, la realidad es muy diferente. El escenario en materia de los derechos humanos pertenecen al reino de lo ideal, la educación en general dista mucho de cumplir con los objetivos propuestos por la política nacional, mientras la educación básica no ha cumplido con la garantía de accesibilidad, asequibilidad y calidad que debe tener; la educación superior por su parte, enfrenta grandes retos para formar a los profesionistas competentes, respetuosos de los derechos humanos y justos que nuestro México requiere, no solo una norma perfecta que incluya el reconocimiento de los derechos fundamentales y la incorporación de los mecanismos mediante los cuales demandar su cumplimiento, se requiere el verdadero cumplimiento de esta protección, en materia educativa, se inscribe tal parece en el deber ser, de la Utopía. (Preciado, 2017)

Hablar de los retos de la educación en México puede significar varias líneas, desde la cobertura y accesibilidad, la calidad, el modelo educativo, las tecnologías, recursos asignados, infraestructura y el propio magisterio, por mencionar. Cada uno de estos retos en nuestros días, conllevan a un análisis minucioso de sus orígenes, características y desafíos muy particulares a la problemática planteada.

En este sentido, y para delimitar el objeto de estudio del presente trabajo, se tomará como referencia lo señalado por el artículo $3^{\circ}$ de la Constitución Política de los Estados Unidos Mexicanos (CPEUM), que reconoce derecho fundamental de toda personal el acceso a la educación, y expresa en su párrafo segundo, las características que esta educación deberá tener: basarse en el respeto irrestricto de la digni- 
dad, con enfoque de derecho humanos e igualdad, tendrá a desarrollar armónicamente las facultades del ser humano y fomentará el amor a la Patricia, respeto a los derechos, libertades, la cultura de paz, la conciencia de la solidaridad internacional, la independencia, la justicia, promoverá la honestidad, los valores y la mejora continua. El cumplimiento de todos y cada uno de los anteriores elementos que debería estar presente en la educación en México, para estar en posibilidad de afirmar que el Estado está cumpliendo con su obligación de proporcionar educación de calidad para su población.

Tomando en cuenta lo anterior nos abocaremos a una sola de las características antes mencionadas de la educación en México: fomentar la cultura de paz. ¿Qué implica esta obligación del Estado Mexicano? ¿Qué debemos entender por educación para la paz? ¿Cuáles son modelos y alcances educativos que permitirán incorporar esta visión de cultura de paz en la educación que se imparta en Jalisco?

Para tal efecto, autores como Virginia Arango Durling, Narcedalia Lozano Garza, José Tuvilla Rayo, Gerardo Pérez Viramontes y el propio Johan Galtung, entre otros autores, serán referentes para contestar las anteriores cuestiones, así como abordar algunas reflexiones entornó a la importancia de la educación desde la perspectiva de cultura de paz, con el objeto de dar respuesta a varias de las grandes necesidades de nuestra sociedad: la tolerancia, el respeto, la resolución pacífica de los conflictos y el respeto a los derechos humanos, principios elementales para transformar la realidad.

La teoría compleja constructiva de la Paz de Johan Galtung, así como las propuestas de modelos educativos para la paz de José Tuvilla Rayo y María del Carmen López, darán sustento teórico para el análisis aquí propuesto. 


\section{Generalidades del contexto}

A partir del año 2000 con la Declaración del Milenio compuesto en ese momento por ocho Objetivos de Desarrollo, se ha buscado la reducción de la pobreza, la consecución de la enseñanza básica universal, objetivos que para el 2015 se complementaron profundizando sus fines, hacia el Desarrollo Sostenible, a partir de cinco grandes grupos: Personas, Planeta, Prosperidad, Paz y Asociaciones. (ONU, En línea)

Si bien, ONU México señaló que para el 2014 se cumplió la meta propuesta en materia de educación, al tener un 95.9\% de niños y niñas que terminaron el ciclo completo de enseñanza primaria (ONU, En Línea), la última estadística del INEGI en 2015, señala que solo un 30.4\% de la población de tres año y más, asisten a la escuela (En línea), si bien identifica que el $93.6 \%$ de la población de 15 años y más en México es alfabeta, lo cierto es que este porcentaje varía en cada entidad federativa, teniendo que en Oaxaca, Chiapas y Guerrero los porcentajes más bajos entre el 84 y 85\% (INEGI, En línea). Y a decir de la CONEVAL, los anteriores datos que ven trastocado el derecho a la educación, tienen que ver con una serie de indicadores como el marco normativo, el presupuesto, la infraestructura, el personal, los planes, programas y materiales de estudio que integran la dimensión de la asequibilidad; los medios físicos y económicos que miden el acceso a la educación; así como la calidad de los medios, de los procesos, el aprovechamiento y relevancia de los contenidos del aprendizaje, lo cual implica la aceptabilidad del derecho a la educación (CONEVAL; 2015, p. 41)

La UNESCO por su parte, reconoce la importancia de la educación como derecho humano y eje transformador de vidas, colocándolo en el centro mismo de su misión "consolidar la paz, erradicar la pobreza e impulsar el desarrollo sostenible” (En línea), en este sentido, coordina la Agenda de Educación Mundial 2030 en el marco del Objetivo de Desarrollo Sostenible 4 (ODS), cuyo objetivo es "garantizar una educa- 
ción inclusiva, equitativa y de calidad y promover las oportunidades de aprendizaje durante toda la vida para todos”.(ONU, En línea)

Lo anterior llevó a la UNESCO a convocar a los diversos organismos internacionales (UNICEF, Banco Mundial, UNFPA, PNUD, ONU Mujeres y ACNUR) al Foro Mundial sobre la Educación 2015, quienes aprobaron la llamada Declaración de Incheon para la Educación 2030, misma que recobra los planteamientos del ODS 4 e incorpora una visión humanista de la educación, basado “...en los derechos humanos y la dignidad, la justicia social, la inclusión, la protección, la diversidad cultural, lingüística y étnica, y la responsabilidad y la rendición de cuentas compartidas." (Marco de Acción Educación (MAE) 2030, 2015, p. iii) Y donde la reconoce además, "esencial para la paz, la tolerancia, la realización humana y el desarrollo sostenible" (MAE 2030, 2015, p. iii) Entre las metas planteadas en esta Declaración, la 4.7, señala:

De aquí a 2030, asegurar que todos los alumnos adquieran los conocimientos teóricos y prácticos necesarios para promover el desarrollo sostenible, entre otras cosas mediante la educación para el desarrollo sostenible y los estilos de vida sostenibles, los derechos humanos, la igualdad entre los géneros, la promoción de una cultura de paz y no violencia, la ciudadanía mundial y la valoración de la diversidad cultural y la contribución de la cultura al desarrollo sostenible. (MAE 2030, 2015, p. 22)

Lo anterior implica una serie de estrategias para cumplir con el objetivo deseado, entre ellas formular políticas programas para promover la educación de desarrollo sostenible (EDS) y la educación para la ciudadanía mundial (ECM); incorporar temas de derechos humanos, igualdad, salud, educación sexual integral, cambio climático, vida sostenible, ciudadanía responsable y participativa; ofrecer una educación para lo largo de la vida, con conocimientos, aptitudes, valores y actitudes para construir sociedades pacíficas, saludables y sostenibles; 
reformar la cooperación y comprensión internacional; así como el compromiso con sus propias comunidades y sociedades; reconocer la cultura como clave para lograr la sostenibilidad y promover el enfoque interdisciplinario que favorezca la EDS y la ECM, a través de los derechos humanos, el fomento de una cultura de paz y no violencia. (MAE, 2015, p. 24)

Sin lugar a dudas el cumplimiento de cada una de las anteriores conlleva a un trabajo de investigación individual, por lo que no se entrará al análisis y discusión de ellos, baste decir que a cuatro años de su promulgación, el Congreso de la Unión aprobaba una nueva reforma educativa constitucional publicada en el Diario Oficial de la Federación (DOF) el 15 de mayo de 2019, la cual además de derogar la anterior reforma del ex presidente Peña Nieto, incorpora entre otras características, el estudio de civismo, cultura, arte, música, deporte y medioambiente; una educación indígena, bilingüe y bicultural, misma que se vio desarrollada mediante la publicación en el Diario Oficial de la Federación del 30 de septiembre de 2019 de la nueva Ley General de Educación.

Por su parte, específicamente en Jalisco se publicó el 15 de mayo de 2020 , la reforma educativa que da cuenta de la disposición del Estado en avanzar al cumplimiento de las metas planteadas por el ODS 4, y donde reconoce el interés superior de niñas, niños y adolescentes como prioridad para la educación que imparta el Estado; reconoce la importancia de los estilos de vida activa y saludables para el proceso educativo y social; así como la cultura de la paz y convivencia democrática para crear entornos escolares libre de violencia.

Lo anterior ha sido un gran avance para el Estado en harás de trabajar en la construcción de una cultura de paz que permita que los educandos, incorporen principios y valores, éticos, morales y democráticos necesarios para la construcción de una ciudadanía activa y responsable para lograr la paz en la sociedad, reconocimiento como 
centro de esta visión, la dignidad humana y el respeto a los derechos humanos.

\section{Marco jurídico}

Marco jurídico nacional

La Constitución Política de los Estados Unidos Mexicanos en su artículo $3^{\circ}$, incorpora como derecho fundamental el derecho de toda persona a la educación. Reconoce la educación básica como obligatoria e impartida por el Estado, priorizando el interés superior de niñas, niños, adolescentes y jóvenes, además de reconocerla como universal, inclusiva, pública, gratuita y laica. Específicamente su párrafo cuarto, señala los elementos es que esta educación se debe basar, a saber.

La educación se basará en el respeto irrestricto de la dignidad de las personas, con un enfoque de derechos humanos y de igualdad sustantiva. Tenderá a desarrollar armónicamente todas las facultades del ser humano y fomentará en él, a la vez, el amor a la Patria, el respeto a todos los derechos, las libertades, la cultura de paz y la conciencia de la solidaridad internacional, en la independencia y en la justicia; promoverá la honestidad, los valores y la mejora continua del proceso de enseñanza aprendizaje.

La anterior garantía se ve reflejada en el desdoblamiento normativo que hace efectiva a la constitución con la Ley General de Educación, reglamentaria del artículo $3^{\text {o }}$ constitucional, que permite el desarrollo de programas y políticas públicas en la materia. Para tal efecto, identifica como sus fines:

I. Contribuir al desarrollo integral y permanente de los educandos

II. Promover el respeto irrestricto de la dignidad humana, como valor fundamental e inalterable de la persona y de la sociedad

III. Inculcar el enfoque de derechos humanos

IV. Fomentar el amor a la Patria 
V. "Formar a los educandos en la cultura de la paz', el respeto, la tolerancia, los valores democráticos que favorezcan el diálogo constructivo, la solidaridad y la búsqueda de acuerdos que permitan la solución no violenta de conflictos y la convivencia en un marco de respeto a las diferencias;"

VI. Propiciar actitudes solidarias en el ámbito internacional

VII. Promover la comprensión, el aprecio, el conocimiento y enseñanza de la pluralidad étnica, cultural y lingüística de la nación

VIII. Inculcar el respeto por la naturaleza, el desarrollo sostenible y la resiliencia frente al cambio climático

IX. Fomentar la honestidad, el civismo y los valores

X. Contribuir al bienestar y desarrollo del país.

Todos y cada uno de los anteriores fines son indispensables para hacer efectivo el derecho a una educación de calidad, pero sobre todo, como señala la fracción V, formar en los educando en la cultura de la paz, visión indispensable para el cumplimiento de los Objetivos de Desarrollo Sostenible.

Ahora bien, para cumplir los anteriores fines, el artículo 30 de la LGE, identifica los contenidos que deberán tener los planes y programas d estudio de la educación que imparta el Estado, entre la que se debe resaltar según la fracción XXI, "La promoción del valor de la justicia, de la observancia de la ley y de la igualdad de las personas ante ésta, la cultura de la legalidad, de la inclusión y la no discriminación, de la paz y la no violencia en cualquier tipo de sus manifestaciones ${ }^{2}$, así como la práctica de los valores y el conocimiento de los derechos humanos para garantizar el respeto a los mismos;", es decir, se identifica como eje transversal en toda la educación básica

1. Negritas propias del autor.

2. Negritas propias del autor. 
la construcción de la cultura de paz y la resolución no violenta de los conflictos.

Por su parte en Jalisco, la Constitución del Estado en su artículo $4^{\text {o }}$ que corresponde a los Derechos Humanos y sus Garantías, incorpora en el apartado B, fracción II el derecho a la educación al señalar: "Garantizar e incrementar los niveles de escolaridad, favoreciendo la educación bilingüe e intercultural, la alfabetización, la conclusión de la educación básica, la capacitación productiva y la educación media superior y superior. Establecer un sistema de becas para los estudiantes indígenas en todos los niveles. Definir y desarrollar programas educativos de contenido regional que reconozcan la herencia cultural de sus pueblos, de acuerdo con las leyes de la materia y en consulta con las comunidades indígenas.", sin embargo es omisa al identificar como un fin primordial para la educación, la visión de cultura de la paz como eje transversal de la formación social.

Lo anterior es paradójico, toda vez que como ya se señaló, el pasado 15 de mayo del año en curso se publicó la nueva Ley de Educación del Estado de Jalisco, la cual replica en su artículo 13 los fines de la educación a que hace referencia la Ley General de Educación antes mencionada, y específicamente en su fracción V reconoce "Formar a los educandos en la cultura de la paz, el respeto, la tolerancia, los valores democráticos que favorezcan el diálogo constructivo, la solidaridad y la búsqueda de acuerdos que permitan la solución no violenta de conflictos y la convivencia en un marco de respeto a las diferencias".

De manera más específica su artículo 82 desarrolla la obligación de todas las autoridades educativas en el ámbito de su competencia de promover la cultura de la paz y la no violencia en las escuelas, con el objetivo de generar una convivencia democrática basada en la dignidad y los derechos humanos, para ello, deberá realizar acciones que involucre tanto a los estudiantes, profesores, padres de familia y personal del plantel educativo en general, que favorezca el sentido de comunidad 
y solidaridad, así como la prevención y atención de la violencia en el entorno familiar. Para ello, entre las acciones que debe cumplir son:

I. Diseñar y aplicar estrategias educativas que generen ambientes basados en una cultura de la paz, para fortalecer la cohesión comunitaria y una convivencia democrática;

II. Incluir en la formación docente contenidos y prácticas relacionados con la cultura de la paz y la resolución pacífica de conflictos;

III. Proporcionar atención psicosocial y, en su caso, orientación sobre las vías legales a la persona agresora y a la víctima de violencia o maltrato escolar, ya sea psicológico, físico o cibernético, así como a las receptoras indirectas de maltrato dentro de las escuelas;

IV. Establecer los mecanismos gratuitos de asesoría, orientación, reporte de casos y de protección para las niñas, niños, adolescentes y jóvenes que estén involucrados en violencia o maltrato escolar, ya sea psicológico, físico o cibernético, procurando ofrecer servicios remotos de atención, a través de una línea pública telefónica u otros medios electrónicos;

V Solicitar a la Comisión Nacional para la Mejora Continua de la Educación estudios, investigaciones, informes y diagnósticos que permitan conocer las causas y la incidencia del fenómeno de violencia o maltrato entre escolares en cualquier tipo, ya sea psicológica, física o cibernética, así como su impacto en el entorno escolar en la deserción de los centros educativos, en el desempeño académico de los educandos, en sus vínculos familiares y comunitarios y el desarrollo integral de todas sus potencialidades, así como las medidas para atender dicha problemática;

VI. Celebrar convenios de cooperación, coordinación y concertación con los sectores públicos, privados y sociales, para promover los derechos de las niñas, niños, adolescentes y jóvenes, y el fomen- 
to de la cultura de la paz, resolución no violenta de conflictos, fortalecimiento de la cohesión comunitaria y convivencia armónica dentro de las escuelas;

VII. Hacer del conocimiento de las autoridades competentes las conductas que pueden resultar constitutivas de infracciones o delitos cometidos en contra de las niñas, los niños, adolescentes y jóvenes por el ejercicio de cualquier maltrato o tipo de violencia en el entorno escolar, familiar o comunitario, así como promover su defensa en las instancias administrativas o judiciales;

VIII. Realizar campañas, mediante el uso de las tecnologías de la información, comunicación, conocimiento y aprendizaje digital, que concienticen sobre la importancia de una convivencia libre de violencia o maltrato, ya sea psicológico, físico o cibernético, en los ámbitos familiar, comunitario, escolar y social; y

IX. Elaborar y difundir materiales educativos para la prevención y atención de los tipos y modalidades de maltrato escolar, así como coordinar campañas de información sobre las mismas.

Es decir, la nueva Ley de Educación tiene muy clara la importancia de la educación para la paz en su sistema educativo: convivencia democrática, resolución no violenta de conflictos, convivencia libre de violencia y su prevención y atención, son temas primordiales para su consolidación, sin embargo, la construcción de una cultura de paz, va más allá de estos indicadores, el combate a las violencias, no debe ser solamente físicos, sino institucionales y culturales como señala Galtung, e incorporar acciones tendientes al cumplimiento de los otros fines de la educación como son: los derechos humanos como eje central; el respeto y tolerancia; la equidad, el medioambiente y el desarrollo sostenible, e incluso la incorporación y uso de las tecnologías, por lo tanto, las anteriores acciones podrían quedar cortas para construir un 
concepto integral de cultura de paz que la educación jalisciense debe proporcionar.

\section{Marco supranacional}

En el marco convencional de los Derechos Humanos, el derecho a la educación y en particular la educación para la paz, cuenta con un amplio sustento en diversos tratados, acuerdos, declaraciones y pactos a los cuales México ha signado su adhesión, y sobre las cuales se hará a continuación.

Empezamos por la Carta de las Naciones Unidas firmada en 1945, señala como sus propósitos en el artículo 1을

1. Mantener la paz y la seguridad internacionales, y con tal fin: tomar medidas colectivas eficaces para prevenir y eliminar amenazas a la paz, y para suprimir actos de agresión u otros quebrantamientos de la paz; y lograr por medios pacíficos, y de conformidad con los principios de la justicia y del derecho internacional, el ajuste o arreglo de controversias o situaciones internacionales susceptibles de conducir a quebrantamientos de la paz;

2. Fomentar entre las naciones relaciones de amistad basadas en el respeto al principio de la igualdad de derechos y al de la libre determinación de los pueblos, y tomar otras medidas adecuadas para fortalecer la paz universal;

3. Realizar la cooperación internacional en la solución de problemas internacionales de carácter económico, social, cultural o humanitario, y en el desarrollo y estímulo del respeto a los derechos humanos y a las libertades fundamentales de todos, sin hacer distinción por motivos de raza, sexo, idioma o religión; y

4. Servir de centro que armonice los esfuerzos de las naciones por alcanzar estos propósitos comunes. (ONU, En línea) 
La Carta anterior, junto con la Declaración Universal de los Derechos Humanos, firmado por México desde el 4 de Noviembre de 1946, son los instrumentos base sobre la cual se sustenta la educación para la paz. El artículo 26 de la Declaración Universal establece el derecho que tiene toda persona a la educación, misma que debe cuando menos a nivel elemental, ser gratuita y obligatoria. Esta educación señala, deberá desarrollar la personalidad humana, fortalecer el respeto y libertad de los derechos humanos, favorecer el respeto y tolerancia a la multiculturalidad, la libertad de religión y la paz. (UNESCO, En Línea)

En el mismo sentido, la Declaración Americana de los Derechos y Deberes del Hombre de 1948, señala en su artículo XII el derecho que tiene toda persona a la educación, la cual cuando menos hasta primaria deberá ser gratuita. Los principios que debe fomentar son los de libertad, moralidad y solidaridad humana; al tiempo que capacite para su subsistencia y lograr un nivel de vida y con ello ser útil a la sociedad. Si bien específicamente no habla de la paz como parte de la educación que debe ofrecer, este derecho a que hace referencia la Declaración se basa en la igualdad de oportunidades que todo ser humano debe tener, de acuerdo a su propia naturaleza. (SCJN, En Línea)

Por su parte, en el Pacto Internacional de Derechos Sociales, Económicos y Culturales, de 1966 y del cual México es parte desde 1981, reconoce en su artículo 13 el derecho de toda persona a la educación. Establece también la obligatoriedad y gratuidad de la enseñanza primaria, e identifica como sus características, desarrollar la personalidad humana, la dignidad y el respeto a los derechos humanos, fomentar la tolerancia, el respeto a los grupos raciales, étnicos y religiosos, así como promover la paz. (Orden Jurídico, En línea)

La convención Internacional para la eliminación de todas las formas de discriminación racial de 1966, al que se adicionó México en 1975, declara el compromiso que adquieren los Estados partes en garantizar el derecho de toda persona a la igualdad y particularmente al goce de 
entre otros derechos al de educación y formación profesional (SRE, En línea), específicamente su artículo 7, compromete la toma de medidas en materia de enseñanza, educación, la cultura y la información, para combatir la discriminación, promover la tolerancia y amistad entre naciones, grupos raciales o étnicos, y propagar los propósitos y principios de la Carta de las Naciones Unidad y la Declaración Universal de Derechos Humanos. (SRE, En línea), propósitos entre los cuales se encuentra precisamente favorecer la paz.

Por su parte la Convención Americana de Derechos Humanos de 1969, adoptada por México en 1981, sobre la educación se limita a reconocer en su artículo 12 el derecho de los padres -y tutores en su caso- a decidir la educación religiosa o moral que sean acordes a sus convicciones. (SCJN, en línea), es decir, no va más allá de la libertad de conciencia y religión como concepción de educación que se debe proteger.

Curiosamente la Convención contra todo tipo de discriminación en la esfera de la enseñanza de 1960, México no ha ratificado, sin embargo es importante mencionar toda vez que reconoce la discriminación en su artículo 1ํo, como toda distinción, exclusión, limitación o preferencia que tenga como objetivo destruir o alterar la igualdad de trato en la enseñanza; o de excluir a una persona o grupo al acceso a diversos grados y tipos de enseñanza.

Señala de igual manera en su artículo $5^{\circ}$, que la educación debe garantizar el pleno desenvolvimiento de la personalidad humana, el respeto de los derechos humanos, las libertades fundamentales, fomentar la comprensión, tolerancia y amistas entre naciones, grupos raciales o religiosos, así como el mantenimiento de la paz. (UNESCO, En línea)

Por su parte, la Convención de los Derechos del Niño de 1980, ratificada por México en el 81, reconoce el derecho de los niños a la educación en igualdad de condiciones. Concretamente, su artículo 29, señala que la educación que reciban deberá estar encaminada a: 
a) Desarrollar la personalidad, las aptitudes y la capacidad mental y física

b) Inculcar al niño el respeto de los derechos humanos y las libertades fundamentales

c) Inculcar al niño el respeto de sus padres, de su propia identidad cultural, de su idioma y sus valores

d) "Preparar al niño para asumir una vida responsable en una sociedad libre, con espíritu de comprensión, paz, tolerancia, igualdad de los sexos y amistad entre todos los pueblos, grupos étnicos, nacionales y religiosos y personas de origen indígena;"

e) Inculcar al niño el respeto del medio ambiente natural. (ONU, en línea)

El Protocolo adicional a la Convención Americana Sobre Derechos Humanos, conocido como "Protocolo de San Salvador", de 1988 y adherido por México en 1996, declara concretamente en su artículo 13 el derecho de toda personal a la educación y establece como fin el pleno desarrollo de la personalidad humana, el sentido de su dignidad y respeto por los derechos humanos, el pluralismo ideológico, las libertades fundamentales, la justicia y la paz. Asimismo, deberá para participar efectivamente en una sociedad democrática y pluralista, lograr su subsistencia digna, así como favorecer la comprensión, la tolerancia y la amistad entre las naciones y los grupos raciales, étnicos o religiosos y el manteamiento de la paz. (SCJN, En línea)

Finalmente, la Declaración de Viena adoptada en el marco de la Conferencia Mundial de los Derechos Humanos en 1993, en materia de educación, señala en el punto 78. "La Conferencia Mundial de Derechos Humanos considera que la educación, la capacitación y la información pública en materia de derechos humanos son indispensables para establecer y promover relaciones estables y armoniosas entre las comunidades y para fomentar la comprensión mutua, la tolerancia y la 
paz." (ONU, En línea), para el cumplimiento de tal fin, se solicita incluir en sus programas de estudio en todas las instituciones de educación, tema sobre derechos humanos, derecho humanitario, democracia e imperio de la ley.

Como se observa el andamiaje supranacional es basto, el derecho internacional da muestra a fondo no solo del reconocimiento e importancia de la educación como derecho humano, sino su valía para garantizarlo al integrar una serie de características que dotará de calidad la educación que el estado tiene obligación de impartir, una de ellas, la construcción de una cultura de la paz desde la educación, es indispensable para el cumplimiento de los objetivos del desarrollo sustentable pero sobre todo, para la formación de una ciudadanía activa, democráticamente responsable, tolerantes y respetuosa de los derechos humanos de todos y cada uno en la sociedad.

\section{Debate actual: la educación como derecho humano para lograr la paz}

Tres grandes temas son hasta ahora el centro de la atención del presente trabajo, Derechos Humanos, Educación y Paz -más concretamente Cultura de Paz-. Todos ellos se pueden analizar de manera independiente, sin embargo, es importante no solo entenderlos en lo individual, sino la conexión y las implicaciones que tienen en su conjunto para lograr una educación de calidad que permita transformar la visión del conflicto que hasta la fecha permea en nuestra sociedad.

Como ya se señaló en el apartado anterior, existe un andamiaje jurídico lo suficientemente abundante para garantizar por parte del Estado su obligación de otorgar educación básica y los elementos y fines que esta debe soportar. Por lo tanto, ahora lo que resta entonces es saber que debe entender por Paz y como Cultura de Paz para conocer hacia donde tiene que nuestra educación. 
Sin lugar a dudas el reconocimiento de la educación como unidad transformadora es importante para el desarrollo social. Actualmente encontramos en el mundo grandes problemas, donde la educación se convierte en el centro para su superación: salud, pobreza, migración, discriminación, contaminación, violencias y delitos; seguridad ciudadana, pública, nacional; terrorismo, corrupción, son algunos de los grandes problemas a nivel global que la sociedad tiene que enfrentar, y para ello la educación es indispensable, no solo por la formación de los diversos profesionistas que las atienden, sino por la preparación de la ciudadanía que les permita conocer la realidad y transformarla en función de los conocimientos adquiridos, es decir, en materia de salud, necesitamos médicos, enfermeras y diversos especialistas que atiendan los problemas de salir que aquejan a nuestro país, pero requerimos de igual manera de ciudadanos responsables que adopten estilos de visa sanos. Por ello, se insiste en que la educación debe ser el eje que permita erradicar muchos de estos problemas.

Ahora bien, como entes sociales, nos encontramos en una búsqueda continua de paz, ya sea personal o colectiva, mental y física, porque identificamos que su concreción se ve afecta por los conflictos, cualquiera que sea su origen. Lamentablemente aquí en donde reside la complejidad de lograr la tan ansiada paz, pues no solo depende de la ausencia de conflictos bélicos cómo se ha manejado durante años, sino que para entender su importancia y lograr incorporarla como un estilo de vida, debemos entender primeramente que no existe una sola paz, y depende de la paz que decidamos adoptar, será la que guie nuestra forma de vida y la manera de manejar los conflictos que en ella aparezcan. Así entonces, empezaremos por definir la paz y la cultura de paz a la que deseamos llegar. 
Galtung y su Teoría de la Paz

Johan Galtung precursor de los estudios de la paz, reconoce en su teoría primeramente la necesidad de identificar el conflicto y la violencia para entender la paz. Para tal efecto, conceptualiza la violencia "como todo agravio evitable a las necesidades humanas básicas y más allá contra la vida rebajando el nivel de satisfacción por debajo de lo potencialmente posible." (Galtung, 1983, en Ramírez, s/a, p. 4), mientras que el conflicto es "...la falta de entendimiento de dos o más partes frente a metas, valores o intereses incompatible ("Galtung, 1996, en Salcedo, 2013, p. 226-227), e identifica una tipología de conflicto a partir de tres violencias: directa; estructural y cultural, cuyos efectos inciden en todos los ámbitos de la vida del ser humano. Esta tipología que se esquematiza por lo regular con un triángulo que permite reconocer la interdependencia que guardan entre sí.

Así encontramos de su exposición, que las violencias directas son las reconocidas como personales y se distinguen por todas aquellas violencias físicas, verbales e incluso psicológicas que comente un ser humano contra otro. Por su parte, las llamadas violencias estructurales, son indirectas, pues se realizan como consecuencia de la omisión y acción de una institución donde no aporta los lineamientos para otorgar las garantías de igualdad, libertad y justicia necesarias para la población y que proceden de estructuras sociales, políticas o económicas arbitrarias. Finalmente, la cultura, que es la menos visible, al ser aquella violencia que dota de simbolismos y genera la educación que a la postre permite que se siga perpetuando las otras dos formas de violencia, caracterizada por los perjuicios, el lenguaje, la ideología y las inequidades, está la observamos en todos nuestros ámbitos sociales: la religión, el arte, la filosofía, la familia, etc.

A partir de lo anterior, identifica cuatro los pilares que componen su teoría de la paz: 1) reconocer el conflicto, 2) resarcir los efectos traumáticos de la violencia 3 ) definir los objetivos de intereses comparti- 
dos y legítimos de las partes para establecer la mediación, y 4) lograr la empatía entre los distanciados por los conflictos, con entendimiento y comprensión. (Galtung, 1990)

Este teórico, hace énfasis en la importancia del fomento de una Cultura de la Paz inclusiva en la que tengan prevalencia los mecanismos pacíficos para resolver un conflicto, una cultura basada en la no violencia, la empatía y la creatividad para transformar el conflicto en los ámbitos individual, social, cultural y político. (Galtung, 1999, en Palacios, 2018) Por ello, su postura es indispensable para transitar hacia la construcción de una cultura de paz desde la educación, pues se conjugan en ella los tres tipos de violencia que debemos erradicar, la directa o personal con la eliminación del bullying, maltratos físicos, verbales o psicológicos, la violencia familiar. La estructural, con el acceso a la educación en igualdad de circunstancias, calidad y seguridad, con la creación en ellas de los mecanismos que permitan y garantices la igualdad, la justicia y la libertad; así como la cultural, con el combate a las ideologías opresivas que promueven el machismo, todo tipo de discriminación y la desigualdad.

Como señala Daissy Liliana Salcedo "La violencia se presenta como una consecuencia de los bajos niveles de desarrollo cuando las condiciones de vida no promueven el bienestar de los individuos y, por el contrario, aumentan su vulnerabilidad." (2013, p. 227), por lo tanto, la educación es indispensable para la construcción de una cultura de paz, bienestar y desarrollo en la sociedad.

\section{De la Paz y la Cultura de la Paz}

El tema de la Paz no es nuevo, como ya se dijo Galtung desde los años ochenta ha desarrollado diversos estudios a su alrededor. Durante los años noventa se caracterizó -entre otras cuestiones- por un proceso de construcción de la paz y de relaciones interpersonales, preocupadas por el ser humano y la reconstrucción de ciudadana, de la política, la 
justicia, así como la transformación estructural y cultural.(Pérez, 2018, p. 8) Se ha transitado desde una visión de erradicar la guerra -conocida como paz negativa- hasta la inclusión de mecanismos que la hagan factible, como son la adopción de medios de resolución de conflictos alternos, la institucionalización de autoridades especializadas, el reconocimiento del respeto a los derechos humanos y la dignidad humana como el centro de su concreción -paz positiva-, hasta la visión de paz imperfecta entendida por Francisco Muñoz como aquella en la cual aceptamos que no solo el Estado tiene injerencia y que en lo individual cada uno de nosotros somos responsables de su concreción día a día sin la perfección que anhelamos. Determinar el tipo de paz que se desea construir es indispensable para construir los mecanismos apropiados para integrar una cultura de paz adecuada.

Así, el constructor de paz es más complejo que solo el deseo de no más guerra, no más inseguridad y violencia, pues como señala Gerardo Pérez Viramontes la paz "Es una idea, un concepto, un valor ... para referirnos a un sinnúmero de situaciones, hechos, sentimientos, momentos o expresiones de nuestra vida en común, a las que generalmente les atribuimos una carga emocional y axiológica positiva..."(2018, p. 78), es decir, esta carga emocional que se le dota al concepto de paz determina el tipo de paz a la que se aspira y condiciona las relaciones y comportamientos de los seres humanos en sociedad.

Pues bien, se entiende por paz desde su origen latino pax, proveniente de la raíz pak, como "resolver algo mediante el acuerdo entre dos partes”. (López Martínez, 2004, en De la Rosa y Cabello, 2016, p. 69), lo que sin lugar a dudas permite identificar los mecanismos de solución de conflictos como parte primordial de toda paz.

Por su parte, Arango Darling Virginia señala que "La paz es uno de los valores máximos de la existencia humana, que está conectado con todos los niveles de aquella, y afecta todas las dimensiones de la vida: 
interpersonal, intergrupal, nacional, internacional y es un proceso dinámico, no estático.” (Jares, 1999, p. 114 en Arango, 2007, p. 15)

Galtung entiende la paz “...como la ausencia de violencia estructural, es decir, como el proceso en el que se logra un estado de armonía del ser humano consigo mismo, con los demás y con la naturaleza." (Galtung, 1996, en Salcedo, 2013, p. 226)

Para Juan Pablo Lederach lejos de entender la paz como un "estado final estático", señala que esta debe concebirse "como una continua evolución y un desarrollo de las relaciones sociales" (Lederach, 2003, p. 11, en Sarti, s/f, p. 4)

Para la UNESCO desde su creación, tiene claro que la paz es un constructo social al reconocer "Que, puesto que las guerras nacen en la mente de los hombres, es en la mente de los hombres donde debe erigirse los baluartes de la paz;" (UNESCO, 2018, p. 7) esta misma declaración, identifica la guerra como resultado de la negación de los principios de dignidad, igualdad y respeto entre los hombres, y reconoce en la cultura y la educación, su valore para lograr la justicia, libertad y paz entre los hombres, misma que debe basarse en la solidaridad intelectual y la moral de la humanidad.

De los anteriores conceptos, se puede entender que más que un derecho, la paz es un deber, un valor, un ideal, una ausencia de cualquier tipo de violencia, una construcción del cual somos todos responsables, y accedemos mediante el reconocimiento y garantía de otros derechos fundamentales, la educación, la libertad, la justicia, la igualdad, donde el objetivo final es el respeto por la dignidad humana. Así entonces, el cumplimiento requerido de una nueva cultura, que permita interiorizar, hacer nuestros y reflejar una conducta de paz en beneficio de la sociedad, pero entonces, ¿Qué se debe entender por cultura de paz?

Para Narcedalia Lozano Garza (2011), la cultura de la paz "es un concepto ilusorio o incluso inexistente", pues significa a su decir "transformar los conflictos, prevenir los conflictos que puedan engendrar 
violencia y restaurar la paz y la confianza en la población que emergen de la guerra. Su propósito transciende los límites de los conflictos armados y se hace extensivo también a las escuelas y a los lugares de trabajo del mundo entero, a los parlamentos y a las salas de prensa, a las familias y a los lugares de recreo." (Lozano, 2011, p. 5)

Por su parte, Gerardo Pérez Viramontes (2018) conceptualiza la cultura de la paz, como "una apuesta ética para hacer frente a esta situación, transmitir el sentido profundo que tiene transformar de forma pacífica los conflictos y exaltar los valores como el conocimiento, el cuidado o la solidaridad." (p. 24)

Al respecto, Emilio Álvarez Icaza y de Imelda González (2017), también se acercan a este concepto al señalar que mediante el mismo, no se busca evitar o eliminar los conflictos, sino adoptar una manera para enfrentarlos, regularlos y resolverlos de forma justa y no violenta. La paz implica identificar las tensiones y las condiciones que favorecen los conflictos, sus secuelas y sus dinámicas.

Finalmente, la Declaración sobre una Cultura de Paz (ONU, 1999), es muy clara al señalar en su artículo 1oㅡ que Cultura de Paz “...es un conjunto de valores, actitudes, tradiciones, comportamiento y estilos de vida basados en:

a) El respeto a la vida, el fin de la violencia, y la promoción y la práctica de la no violencia por medio de la educación, el diálogo y la cooperación;

b) El respeto pleno de los principios de soberanía, integridad territorial e independencia política de los Estado y de no injerencia en los asuntos que son esencialmente jurisdicción interna de los Estados, de conformidad con la Carta de las Naciones Unidas y el derecho internacional;

c) El respeto pleno y la promoción de todos los derechos humanos y las libertades fundamentales;

d) El compromiso con el arreglo pacífico de los conflictos; 
e) Los esfuerzos para satisfacer las necesidades de desarrollo y protección del medio ambiente de las generaciones presente y futuras;

f) El respeto y la promoción del derecho al desarrollo;

g) El respeto y el fomento de la igualdad de derechos y oportunidades de mujeres y hombres;

d) El respeto y el fomento del derecho de todas las formas a la libertad de expresión, opinión e información;

e) La adhesión a los principios de libertad, justicia, democracia, tolerancia, solidaridad, cooperación, pluralismo, diversidad cultural, diálogo y entendimiento a todos los niveles de la sociedad y entre las naciones;

f) Y animados por un entorno nacional e internacional que favorezca a la paz".

En esta misma fecha, la Asamblea General de las Naciones Unidad, emite el Programa de Acción sobre una Cultura de Paz (1999), las siguientes líneas para lograr las metas planteadas en los anteriores principios:

1. Educación para la paz

2. Desarrollo económico social y cultural

3. Desarrollo ambiental sostenible

4. Derechos Humanos

5. Igualdad y no discriminación

6. Gobernanza y participación democrática

7. Respeto y solidaridad

8. Innovación, información y conocimiento

9. Manejo de conflictos y seguridad.

Es decir, la construcción de una cultura de paz, es un proceso más complejo que la simple visión de lograr la erradicación de la guerra, los conflictos armados, el terrorismo o la seguridad ciudadana más en 
lo local, su implementación implica un ejercicio por todos los actores sociales e institucionales que llevarán al combate con las tres formas de violencia que Galtung ha identificado.

La educación para la paz, es tan solo uno de los engranajes para su aseguramiento, así como el respeto, protección y garantía de los derechos humanos, acciones que sin lugar a dudas van de la mano.

\section{Educación para la paz, modelos y oportunidades}

Finalizada la Segunda Guerra Mundial, surge un segundo periodo del comienzo de la educación para la paz; con la influencia filosófica de los estudios concebidos a partir de la llamada investigación para la paz -Peace Research-, de su referente teórico más importante, Johan Galtung, quien ha percibido la paz desde: "interdisciplinariedad, transdisciplinariedad, carácter normativo, orientación hacia la acción, naturaleza internacional, receptores diversos, etcétera" (Asociación Pro Derechos Humanos, 1994, en Cabello Et al, 2016, p. 95)

La educación es una herramienta indispensable para generar un desarrollo democrático participativo, equitativo tanto social como personal, la consolidación de una ciudadanía crítica y activa (Salcedo, 2013) y con ello, renovar la cultura, la forma de pensar y de actuar, a través del respeto y ejercicio del derecho humano a la paz. (Tuvilla, s/f, p. 2)

La educación como derecho fundamental, es la base para el desarrollo humano, para el crecimiento social, económico y cultural; su incremento se reflejará en la disminución de la violencia y el conflicto en la sociedad, de la desigualdad, la pobreza, mejorando las condiciones de vida, del trabajo conjunto, del diálogo y la construcción de nuevos mecanismos de solución de sus conflictos. En virtud de ello, la paz y específicamente la educación para la paz se convierte en una herramienta indispensable para crear ciudadanía "activos, críticos, reflexivos y abiertos a la diversidad religiosa, cultural, social, étnica o política” (Nussbaum, 2010, en Salcedo, 2013, p. 231-232) 
Así entonces, la educación para la paz es un proceso educativo que se fundamenta en los principios de la paz positiva, para fomentar una nueva cultura donde el conflicto se encuentra presente y se enseña a la sociedad a entender su realidad de manera crítica para su solución. (Cabello et al., 2016)

Para Pérez Viramontes, la educación para la paz “es una práctica social que promueve ciertos valores, trata de hacer emerger un tipo determinado de emociones (empatía, afecto, cuidado, respeto...) e impulsa la resignificación de palabras y conceptos con lo que nos relacionamos. (2018, p. 82), por lo tanto, al ser una práctica social, se entiende que es una acción colectiva, donde el esfuerzo para transformar la realidad y satisfacer las necesidades debe ser desde la visión de comunidad.

Como se ha visto, la educación para la paz se encuentra fundamentada en los instrumentos internacionales que ya se han señalado en el apartado correspondiente, más aún, necesarios para cumplir con la visión humanista de la educación que establece la Declaración de Incheon para la Educación 2030. Parte de la educación para la paz, es precisamente formación de la sociedad desde una visión global, es decir, de desarrollo internacional. Para ello, la solidaridad, la tolerancia y el respecto son principios básicos en la protección, conocimiento y enseñanza de derechos humanos como parte de la pedagogía para la paz.

Como señala Tuvilla Rayo, "La Cultura de Paz como ha quedado demostrado constituye en la actualidad la finalidad de la educación, dado que la paz es un concepto síntesis que engloba la interdependencia entre todos los derechos humanos, de manera que estos constituyen los principios, fines y objetivos del derecho a la educación." (Tuvilla, s/f, p. 36), por lo tanto, este autor propone incorporar el modelo educativo que permita cumplir con la visión de paz que se pretende implantar en la sociedad. 
Modelos de educación para la cultura de paz

Las últimas reformas educativas tanto naciones como locales, deberán poner en marcha una estrategia y diseño del modelo educativo que permita formar a la sociedad en el tipo de Paz que se quiere fomentar en la población, una educación basada en derechos humanos, democracia, equidad, justicia para toda la comunidad, o cómo se identificó desde el Grupo de Lisboa (1995) las cuatro "D” necesarias para lograr una Cultura de Paz: derechos humanos, democracia, desarme y desarrollo humano sostenible.

Para tal efecto, José Tuvilla Rayo (s/f) presenta dos tipos de modelos educativos que permiten entender cómo construir un programa de educación para la paz adecuado al contexto nacional o internacional al cual se quiera aplicar.

a) Modelos de programas internacionales (Tuvilla, s/f, 37-39).

- Modelos o enfoques restringidos: Cuya principal característica es la de programas contenidos de derechos humanos y de la paz, desde el conocimiento de los principios constitucionales y valores democráticos.

- Modelos o enfoques extensivos: Donde los programas tienen como eje central la Educación para la Paz, con objetivos y contenidos transversales que abonan hacia la transformación y construcción de la justicia social y no solo para conocer los principios y valores democráticos.

- Modelos o enfoques integrales: Caracterizado por una concepción de educación global o mundial, cuya visión holística de la educación para la paz pretende construir una Cultura de Paz, desde la visión de una nueva ciudadanía, respetuosa de los derechos humano, la democracia, la tolerancia, el desarrollo y el medioambiente. Los contenidos se abordan no solo desde unidades de aprendizaje aisladas, 
sino mediante una nueva metodología de estudio que armoniza los conocimientos de la ciencia, la cultura y la comunicación.

b) Modelos de programas regionales y nacionales (Tuvilla, s/f, pp. 3839).

- Modelo de Educación en Valores: Su principal característica es la de incorporar una enseñanza de derechos humanos y paz, desde la democracia y la ciudadana como valores públicos.

- Modelo de Educación para la Paz y los Derechos Humanos, protectora y promotora de derechos y valores: Cuyos programas van dirigidos más que el cambio personal, al cambio social, por lo que este modelo conocidos también como de "empoderamiento", va dirigido hacia la protección de los grupos más vulnerables mediante la capacitación agentes clave como activistas, profesionales, autoridades y responsables políticos.

- Modelo de Educación para la Cultura de Paz como motor de transformación: Tiene una gran conexión con los problemas mundiales y entre sus objetivos se encuentra la promoción y protección de los derechos humanos con programas institucionales que se desarrollan desde la educación formal y no formal.

Aunados a los anteriores modelos, Gerardo Pérez Viramontes (2018, pp. 120-121) hace referencia a otros modelos curriculares desarrollados por María Carmen López López (1997), que contribuyen a construir paz a partir del contexto escolar o el nivel educativo en el que se trabaja, a los cuales se les denominará local o contextual para seguir con la línea trazada por Tuvilla Rayo anteriormente:

c) Modelos de programas locales o contextuales

- Modelo técnico-instrumental: Que parte de una visión de Educación de Paz desde la concepción negativa de la paz. Pretende sensibilizar a los estudiantes sobre la importancia de la ausencia de guerras y violencias directas, favoreciendo programas, cursos o laborato- 
rios independientes que aboguen por la armonía, la estabilidad y el equilibrio para evitar los conflictos.

- Modelo interpretativo-cultural: Su enseñanza se centra en una perspectiva psicológica y sociocultural. Le da significado a las razones, intensiones y significados simbólicos que se van dando en la sociedad a partir de su interacción, por lo que sus contenidos no son preestablecidos, sino que se van construyendo por el profesor -visto como agente de cambio- en razón de cada contexto escolar, de cada interés del estudiante, para dar vida al aprendizaje y las relaciones en el aula, e independiente al contexto social, político y económico que rodea a la escuela.

- Modelo socio-crítico: Su objetivo es la transformación de la realidad, a partir de la crítica social y donde la educación para la paz se incluye desde una visión conjunta de la política, la económica y la social. Centra su aprendizaje en el estudiante como agente transformador, y toma en cuenta el contexto social, institucional y personal para ello. El profesor es visto como un profesional con la capacidad de regular y transformar el conflicto e incluir un aprendizaje y disciplina colectiva. Así, demanda en el aprendizaje de una ética individual y social muy arraigada, que permita como de la estructura social; intenta derribar los discursos de violencia estructural y cultural que se susciten para transformas las estructuras injustas y paralizadas.

Independientemente del modelo que se adopte para estructurar una verdadera educación para la paz, lo que es evidente es entender que este es un proceso complejo y no aislado, que debe incluir una visión multicultural, transversal y global, donde el respeto, la tolerancia, la equidad y el pensamiento crítico deben ser parte esencial de la formación para inculcar una nueva forma de vivir en sociedad, en contraposición a educar sobre la paz que implica solamente una transmisión de conceptos sin estructura ni objetivos, educar para la paz presupone “... 
no solo informar sobre la amplia cosmovisión de la paz sino que paralelamente exige un replanteamiento del propio proceso de enseñanza -aprendizaje, acorde con los valores de la paz" (Jares, 1997, p. 120 en Pérez, 2018, p. 86) Enseñar la paz implica un cambió de cosmovisión, pues para enseñar la paz hay que vivir en paz.

\section{Conclusiones}

Pese a los trabajos que los gobiernos han desarrollado los últimos veinte años para reducir la pobreza y garantizar el acceso a la educación básica de conformidad con las metas planteadas en los Objetivos de Desarrollo, lo cierto es que aún existen grandes rezagos, en accesibilidad, equidad y calidad de la educación, que permita transformar la vida de la sociedad. Como agente para erradicar la pobreza, impulsar el desarrollo así como consolidar la paz, el sistema de educación en el país muestra aún atrasos, tal como lo muestra el INEGI, sobre todo para las entidades federativas donde se observan grandes problemas de pobreza, violencia e inseguridad.

Quedan diez años para cumplir la meta al 2030 e incorporar una visión humanista de la educación de conformidad con la Declaración de Incheon, cuyo objetivo debe ser la construcción de una cultura de paz que permita el desarrollo social y sostenible, ello implica la inclusión no solo de temas de derechos humanos, tolerancia y respeto, sino la edificación de un nuevo modelo de educación para la paz para toda la vida, que redefina a la ciudadanía y que incorpore una visión global de la paz desde la multiculturalidad, la democracia, el cuidado del medioambiente y el desarrollo sostenible como principios indispensables.

Mientras la Constitución Federal sustenta el derecho fundamental para acceder a una educación que fomente entre otras características la cultura de paz, la Constitución Local es omisa al respecto, es decir, 
reconoce en su artículo 4to el derecho a la educación, sin embargo no incluye la cultura de la paz como uno de sus elementos, aún que ley secundaria local en su última reforma sí busca la garantizar la constitucionalidad del derecho a la educación para la paz, en el sentido de formar en la cultura de paz, el respeto, la tolerancia, valores democráticos, la solidaridad y la solución pacífica de los conflictos, para lo cual, esta educación deberá promover la justicia, la igualdad, la legalidad, la inclusión y no discriminación, la no violencia en cualquiera de sus manifestaciones, la práctica de valores y el respeto de los derechos humanos.

Para el cumplimiento de lo anterior, la nueva Ley de Educación de Jalisco, tiene claro las acciones a desarrollar para incorporar una educación para la paz en el sistema educativo, a partir de la convivencia democrática, resolución no violenta de conflictos, convivencia libre de violencia, su prevención y atención, el sentido de comunidad y solidaridad, todo ello basado en el respeto de los derechos humanos.

A nivel supranacional, se observa cuatro momentos en la inclusión de educación para la paz, después de la segunda guerra mundial, los instrumentos internacionales señalan de manera muy general la educación como derecho humano especificando en algunos casos que ésta debe entre otras cuestiones favorecer o promover la paz. A partir de los años sesenta se observa un segundo momento donde se amplía esta característica en la educación incluyendo término morales sobre igualdad, discriminación y tolerancia; es a partir de los años ochenta que se incluye además de la paz como característica en la educación, la visión de derechos humanos y libertades, dignidad, identidad cultural, respeto al medioambiente, pluralismo ideológico y democracia, constituyéndose con ello un tercer momento en la educación sobre la paz, más que para la paz.

Finalmente, es a partir de los Objetivos del Milenio y ahora Objetivos de Desarrollo Sostenible, se reconoce la importancia de la educa- 
ción para la paz durante toda la vida, la cual deberá de ser humanista, inclusiva, equitativa, que permita la realización humana y el desarrollo sostenible, que promueva la cultura de paz y conforme una ciudadanía global.

Educar para la paz en Jalisco constituye un gran reto, pues la educación se tiene que dejar de ver como un simple proceso de enseñanza aprendizaje, donde la transmisión de conocimientos es el único objetivo, hay que educar para toda la vida, no solo en el sentido de enseñar a aprender, sino de enseñar a vivir, a sentir, a convivir en sociedad, a crear comunidad, ciudadanía y hermandad, es decir a coexistir en paz con uno mismo y con la sociedad en general. Para ello, la dignidad, los derechos humanos, la democracia y el desarrollo sostenible (el aprendizaje de las $4 \mathrm{D}$ ) son indispensables, pero siempre bajo los principios de solidaridad, respeto, tolerancia, igualdad y justicia.

Lo anterior conlleva el diseño de planes y programas de estudio donde la visión de Cultura de Paz se incorpore de manera transversal, no como un contenido sino como un enfoque, para tal efecto, escoger un solo modelo seria limitado toda vez que la construcción de paz debe de ser global. Así, de los modelos internacionales se debe apostar por una perspectiva integral que incluya una metodología que armonice los conocimientos, los principios y los valores de una cultura de paz mundial. Esto va de la mano a modelo nacional que concibe la Cultura de Paz como motor de transformación, por lo tanto, esta educación que se imparta no es solo responsabilidad del Estado, se debe incorporar en todos los ámbitos de la sociedad, en toda la educación formal y no formal que se realice y en todos los niveles educativos.

Por último, hay que adaptar los anteriores modelos hacia lo local, donde para tal efecto el modelo socio-critico, permitirá incluir en los programas de estudios esta visión transformadora de la realidad, mediante un sistema de enseñanza aprendizaje basado no solo en conocimientos aislados, sino en un pensamiento crítico que permita a los 
estudiantes ser verdaderos agentes del cambio personal, social y ambiental.

\section{Bibliografía}

Álvarez Icaza, E. y González, I. (2017). Derechos humanos, ciudadanía y paz. Construcción de la democracia en México. México: Cátedra

Animal Político (1o Mayo 2019) Estos son los puntos principales de la reforma educativa. México. En línea, consultado el 17 de junio de 2020 en: https://www. animalpolitico.com/2019/05/puntos-iniciativa-reforma-educativa/

Cabello Tijerina, Paris Alejandro Et al, (2016) "Cultura de Paz”, México: Universidad Autónoma de Nuevo León.

Consejo Nacional de Evaluación de la Política de Desarrollo Social, (CONEVAL 2018) “Estudio Diagnóstico del Derecho a la Educación 2018", Ciudad de México. En línea, consultado el 16 de junio de 2020 en: https://www.coneval.org.mx/ Evaluacion/IEPSM/Documents/Derechos_Sociales/Estudio_Diag_Edu_2018. pdf

Convención Americana de Derecho Humanos, Suprema Corte de Justicia de la Nación, consultado el 17 de septiembre de 2013, en: http://legislacion.scjn.gob. $\mathrm{mx} /$ TratadosInternacionales/Reformas.aspx?IdLey=22843

Convención Internacional para la Eliminación de Todas las Formas de Discriminación Racial, Secretaria de Relaciones Exteriores, consultado el 13 de septiembre de 2013, en: http://www.sre.gob.mx/images/stories/dgpme/acuerdos/ coninter_eliminar_formasdiscriminacion.pdf

Convención para la Eliminación de todas las Formas de Discriminación contra la Mujer, Instituto de las Mujeres, consultado el 13 de septiembre de 2013 en: www.edoc.inmujeres.gob.mx/documentos_download/100039.pdf

Convención de los Derechos del Niño, Organización de las Naciones Unidas, consultado el día 13 de septiembre de 2013, en: http://www.ohchr.org/SP/ProfessionalInterest/Pages/CRC.aspx 
Convención contra todo tipo de Discriminación en la Esfera de la Enseñanza, UNESCO, consultado el 13 de septiembre de 2013, en: http://portal.unesco.org/ es/ev.phpURL_ID=12949\&URL_DO=DO_TOPIC\&URL_SECTION=201.html

Declaración Americana de los Derechos y Deberes del Hombre, Suprema Corte de Justicia de la Nación, consultado el 17 de septiembre de 2013 en: www.legislacion.scjn.gob.mx/TratadosInternacionales/Reformas.aspx?IdLey=89694

Declaración Universal de los Derechos Humanos, UNESCO, consultado el 13 de septiembre de 2013, en: http://unesdoc.unesco.org/images/oo17/oo1790/179018m. pdf

Declaración y Programa de Acción de Viena, (1993) “Conferencia Mundial de los Derechos Humanos”, Viena, ONU. En línea, consultado el 20 de junio de 2020, en: https://www.ohchr.org/Documents/Events/OHCHR2o/VDPA_booklet_ Spanish.pdf

De la Rosa Vázquez, Cecilia Sarahí y Cabello Tijerina, París Alejandro (2017) "Análisis de la tipología de la paz del siglo XXI", en Revista Pensamiento Americano, Vol. 10, Issue 19, julio - diciembre, pp. 68 - 79. Colombia: Corporación Universitaria Americana. Consultado en línea el 03 de julio de 2019 en: https://www. redib.org/recursos/Record/oai_articulo1471609-ana\%CC\%81lisis-tipologi\%CC\%81a-paz-siglo-xxi

Galtung, J. (Aug. 1990) “Cultural Violence”, publicado en el Journal of Peace Research, Vol. 27, No. 3, pp. 291-305.

El Informador (6 julio 2019) "Presentan nuevo modelo educativo”. México. En línea, consultado del 17 de junio de 2020 en: https://www.informador.mx/jalisco/Presentan-nuevo-modelo-educativo-para-Jalisco-20190706-oo7o.html

INEGI, (s/f) “Analfabetismo", Cuéntame...población. En línea, consultado el 16 de junio de 2016 en: http://cuentame.inegi.org.mx/poblacion/analfabeta.aspx?te$\mathrm{ma}=\mathrm{P}$

Lederach, John Paul (1998) Construyendo la paz. Reconciliación sostenible en sociedades divididas. Bilbao: Bakeaz-Gernika Gogoratuz.

Lozano Garza, Narcedalia (2011) De Teorías, Metodologías y Prácticas para la paz. "VI Jornadas de jóvenes investigadores.” Instituto de Investigaciones Gino 
Germani, Facultad de Ciencias Sociales, Buenos Aires: Universidad de Buenos Aires.

ONU México (s/f), Objetivos de Desarrollo del Milenio. En línea, consultado el 16 de junio de 2020 en: http://www.onu.org.mx/agenda-2030/objetivos-de-desarrollo-del-milenio/

Pacto Internacional de Derechos Sociales, Económicos y Culturales, Orden Jurídico Nacional, consultado el 13 de septiembre de 2013 en: http://www.ordenjuridico.gob.mx/TratInt/Derechos\%20Humanos/D50.pdf

Palacios, A. (2018) “Tras la violencia, las tres erres de Galtung: reconstrucción, reconciliación y resolución”. Consultado en línea el 22 de agosto de 2018 en https://www.unav.edu/web/global-affairs/detalle/-/blogs/tras-la-violencia-las-tres-erres-de-galtung-reconstruccion-reconciliacion-y-resolucion

Pérez Viramontes, Gerardo (2018) "Construir paz y transformar conflictos. Algunas claves desde la investigación, la investigación y la cultura de paz”. Jalisco: ITESO.

Preciado Rodríguez, Teresa Magnolia (2017) “Del derecho a la educación, a la enseñanza basada en los derechos humanos", Revista Derechos Fundamentales a Debate, No. 4, abril-julio, México, Comisión Estatal de Derechos Humanos Jalisco, pp. $51-57$.

Protocolo Adicional a la Convención Americana sobre Derechos Humanos en materia de Derechos Económicos, Sociales y Culturales "Protocolo de San Salvador", Suprema Corte de Justicia de la Nación, consultado el 17 de septiembre de 2013, en: http://legislacion.scjn.gob.mx/TratadosInternacionales/ Reformas.aspx?IdLey=89235

UNESCO, (2015) “Educación 2030. Declaración de Incheon y Marco de Acción Hacia una educación inclusiva y equitativa de calidad y un aprendiza a los largo de la vida para otros.” Incheón, 2015.

UNESCO, (2018) “Textos Fundamentales: Constitución de la Organización de las Naciones Unidas para la Educación, la Ciencia y la Cultura”. Paris. Consultado el 22 de junio de 2020 en: https://unesdoc.unesco.org/ark:/48223/pfoooo261751_ spa.page $=7$ 
Ramírez Martínez, Daniel Fernando "Elementos a solucionar para llegar a una paz positiva, una mirada desde la Teoría de Johan Galtung”, (S/F) Consultado el 2 de noviembre de 2019 en: http://media.utp.edu.co/vicerrectoria-de-investigaciones/archivos/PONENCIA\%20-\%20ELEMENTOS\%20A\%20SOLUCIONAR\%20PARA\%2oLLEGAR\%20A\%20UNA\%2oPAZ.pdf

Salcedo, Daissy Liliana (2013) "En el camino al desarrollo. La educación como medio para la construcción de paz", en Observatorio de Construcción de Paz, Democracia, desarrollo y construcción de paz. Serie documentos para la paz No. 4, Colombia: Universidad de Bogotá Jorge Tadeo Lozano, pp. 223-248.

Tuvilla Rayo, José (s/f) "El Derecho Humano a la Paz en la Educación: Construir la Cultura de Paz”. Consultado en línea el 23 de junio de 2020 en: https:// www.academia.edu/12369151/HUMANO_A_LA_PAZ_EN_LA_EDUCACI\%C3\%93N_CONSTRUIR_LA_CULTURA_DE_PAZ 